\title{
LOW-ABERRATION IMAGING MONOCHROMATOR WITH DISPERSION SUBTRACTION BASED ON AN AXIALLY SYMMETRIC DESIGN
}

\author{
I. M. Gulis ${ }^{*}$ and A. G. Kupreyeu
}

UDC 681.785 .554

\begin{abstract}
An axially symmetric optical system for an imaging monochromator with dispersion subtraction for multiple multispectral scans is proposed and simulated numerically. This system provides minimal angles of incidence for beams on spherical objectives so that off-axis aberrations are reduced over the entire image plane. The width of the selected spectral band for individual image segments is independent of the entrance aperture size. A method of eliminating background illumination of the detector is proposed and the light losses are estimated.
\end{abstract}

Keywords: multispectral scan, dispersive imaging monochromator.

Introduction. Spatially resolved spectroscopy (SRS) provides spectral information on extended (generally, twodimensional) regions of space. The recorded hyperspectral image (spectral hypercube, referred to as the hypercube in the following) $I(x, y, \lambda)$ matches each quasipoint element $(x, y)$ of the region under study to its spectral characteristic $I(\lambda)$. Originally SRS was used in geology, but is now used in ecology, agriculture [1,2], industry, monitoring urban infrastructure [3], and other applications [4].

The volume of data and the rate at which it is acquired, as well as the feasibility of scanning the entire region of study at a single time [5], depend on the hypercube detection technique. With slit scanning (pushbroom), spectral data from a narrow entrance slit is imaged simultaneously on a two-dimensional array - the cross section of the hypercube on the spectral axis and along one spatial coordinate $[6,7]$. Here a spectral resolution comparable to that provided by ordinary dispersion spectrometers can be attained, but it is not possible simultaneously to image the object within a narrow spectral interval; the spatial and spectral resolution depend on the slit width. With narrow-band spectral filtering, the hypercube is detected as a set of cross sections along the spatial coordinates - narrow-band images of the region being studied. Using interchangeable optical band filters or tunable narrow-band (acousto- and electro-optical, interference polarization) filters for scanning along the spectral coordinate does not simultaneously provide a broad spectral working range with a high spectral resolution and optical throughput; the background outside a nominal spectral band is not adequately suppressed [8]. Simultaneous detection of the complete hypercube generally assumes scanning of its cross sections on a two-dimensional or multilayer photodetector [9-12]. However, the pixellation of the photodetector and aberrations during operation over an extended field impose limits on the number of cross sections that can be detected and prevent simultaneous high spatial and spectral resolution.

The major tasks for SRS (surface evaluation, detection of anomalies, and target recognition) require identification of small spatial regions with characteristic spectral components; that is, it is important to have high spatial resolution over the entire image plane. Less strict requirements are imposed on the spectral resolution, at a level of tens of nanometers (the modern multispectral sensors of the Landsat-8 satellite record 20-75 $\mathrm{nm}$ bands in the visible [13]). This resolution is sufficient for a wide range of monitoring tasks on the earth's surface, monitoring of production, and medical applications. In these applications the spectral characteristics of objects are determined by the fundamental mechanisms for broadening of the electronic absorption and emission bands of centers in condensed media, which lead to typical widths $>30-50 \mathrm{~nm}$ for individual structural elements of the spectra. (Higher spectral resolution may be required for taking the spectra of gaseous components of the atmosphere and astronomical objects.) Under these conditions, narrow-band spectral filtration is the

* To whom correspondence should be addressed.

Belarusian State University, 4 Nezavisimost' Ave., Minsk, 220030, Belarus; e-mail: gulis@bsu.by. Translated from Zhurnal Prikladnoi Spektroskopii, Vol. 82, No. 1, pp. 135-140, January-February, 2015. Original article submitted September 30, 2014. 


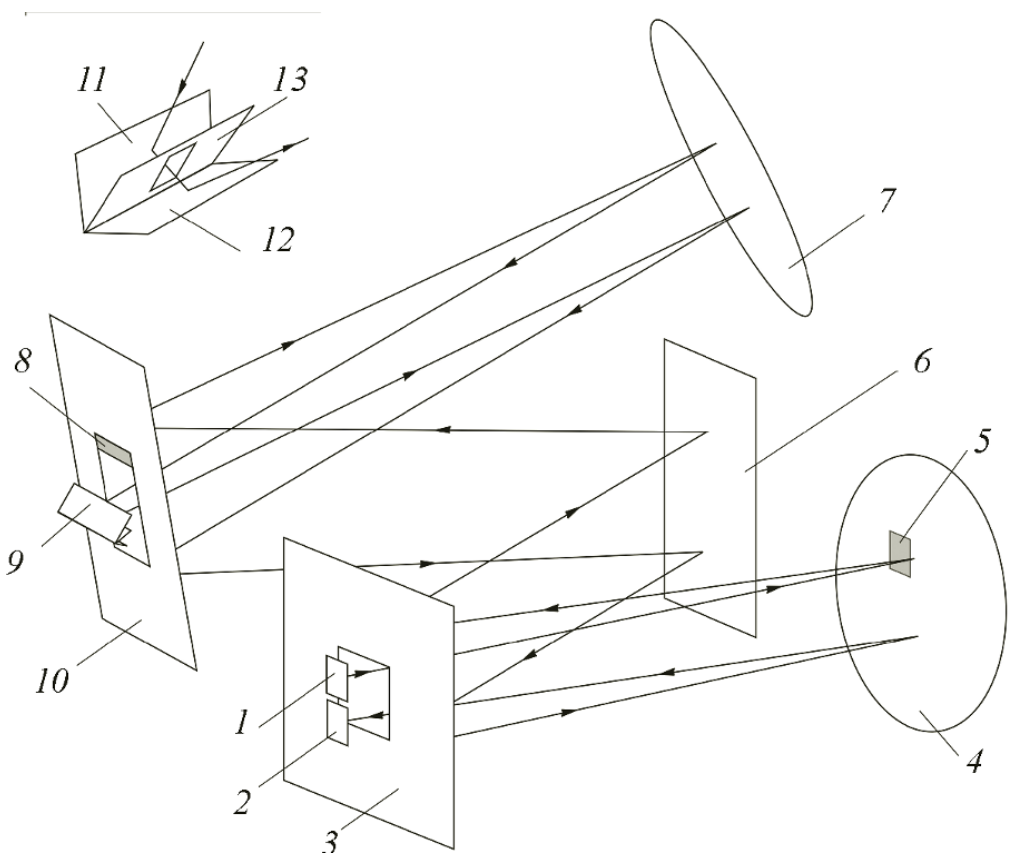

Fig. 1. Optical configuration of a dispersing imaging monochromator with dispersion subtraction and an illustration of the propagation of a light beam through it (indicated by arrows): (1) entrance aperture, $(2)$ detector, $(3,10)$ plane mirrors with aperture stops, (4) collimating mirror objective, $(5,8)$ light-absorbing screens, (6) diffraction grating, (7) camera mirror objective, (9) mirror reflector with a slit mask; the inset shows a mirror reflector with a slit mask (11, 12 plane mirrors; 13 mask with aperture).

optimum way of hypercube scanning. The possibility of simultaneously recording the entire observable region within a specified spectral range makes it possible to introduce operational monitoring.

By using narrow-band spectral filtration of devices based on dispersing monochromators (imaging monochromators) it might be possible to obtain high spectral resolution and efficient background suppression at wavelengths outside a given band; this is an advantage over systems based on band or tunable filters. (This last point is especially important for small spectral band widths.) The difference from dispersing devices with slit scanning is that the spectral and spatial resolutions are independent, so it is possible to widen the entrance aperture and simultaneously record an extended region in a narrow spectral range. At the same time, the imaging characteristics of this sort of system must meet more demanding specifications.

A method has been proposed for obtaining narrow-band spectral images using a double monochromator with dispersion subtraction. The wide entrance aperture of the instrument forms a set of chromatic images that are shifted relative to one another in the image plane of the first monochromator. A slit mask is used to isolate a segment from this pattern that corresponds to the set of images of the aperture for wavelengths in a chosen spectral band. This segment is fed to the entrance of the second monochromator with the dispersion subtracted to form an image of the entrance aperture without spectral "smearing." The distinctive feature of this configuration is the subtraction of dispersion by reverse passage of the beam isolated by the mask through the same optical system; this automatically ensures the required identity of the two monochromators and makes it possible to scan over the spectral range by turning a single dispersing element. The light beam is turned symmetrically for reversed passage by two plane mirrors mounted like a corner reflector so that an intermediate image is formed in their bisector plane (where the slit mask lies). It has been shown [15] that the spectral resolution for the individual image segments in this approach is independent of the size of the entrance aperture but is determined by the width of the slit mask. The spatial resolution is limited by the aberration characteristics of the imaging system and the detector pixel size.

Numerical simulations for a scheme close to the Czerny-Turner configuration indicates that aberrations are present, primarily astigmatism and coma owing to the large numerical aperture and oblique incident of the light beam on the mirror objectives. The known ways of minimizing them [16] are based on using aspheric objectives and complicated diffraction 
gratings (convex, with variable periods) or introducing supplementary optical elements for correcting the light field, which makes the system more complicated and costly.

Constructing a system for axially symmetric passage of the beams through the objectives can greatly reduce the level of aberration without making the optical layout more complicated. Several basic design variants have been examined; the simplest and technically realizable is a system [17] in which the light beam enters and leaves the polychromator (an important problem for this kind of layout) through plane mirrors with aperture stops.

The optical configuration of an imaging monochromator with reverse passage and dispersion subtraction is shown in Fig. 1. A light beam from the entrance aperture 1 is collimated by an objective 4; after reflection from a plane mirror 3 it is incident on a diffraction grating 6 . The dispersed components are directed to an objective 7 by a plane mirror 10, which forms a set of chromatic images of the aperture in the plane of a mask 13. The chromatic light beams isolated by the mask proceed symmetrically to a direct path and, after the dispersion of the grating 6 is subtracted, are combined at the detector 2 .

We have carried out numerical simulations to evaluate the imaging characteristics and optical throughput of this configuration taking losses at the aperture stops into account. The choice of system parameters is determined by a balance between optical throughput and spatial resolution, on one hand, and size, on the other.

Features of the Optical Configuration. The operation of an optical system with a 1:1 magnification was evaluated; thus, the entrance 1 and exit 2 apertures are the same in size, while the collimator 4 and camera 7 objectives have the same focal distance. The two apertures have sizes of $7.18 \times 5.32 \mathrm{~mm}$, which correspond to a commercially available series of photodetector arrays with a diagonal of $1 / 1.8 "$. The apertures are positioned symmetrically in different half spaces relative to the plane of symmetry (the dispersion plane), but lie in a single plane. The distance from the symmetry plane to the nearest side of each of them is minimized in order to reduce off-axis aberrations and equals $1.33 \mathrm{~mm}$.

A larger focal distance for fixed apertures improves the image quality over the field by reducing its angular size, but then the linear dimensions of the system are increased and the dimensions of the mirror objects are much larger. The focal distance of objectives 4 and 7 is $333.5 \mathrm{~mm}$ (radius of curvature $667 \mathrm{~mm}$ ), so that the linear dimensions of the objective 7 do not exceed $150 \mathrm{~mm}$.

A 1200 lines/mm diffraction grating is used as the dispersing element. The angle between mirrors 11 and 12 of the reflector is $87.8^{\circ}$, so that after reflection from the first mirror the central ray of the beam from the center of the aperture is perpendicular to the plane of the bisector, and the intermediate image is formed near it. Because of this configuration the reverse propagation is symmetric to the direct path for the axial beams.

As a rule in configurations with axial propagation, the light beams enter the system and leave for the detector through aperture stops in the optical elements or in supplementary plane mirrors. With other conditions the same, the optical throughput in the second case is lower: in order to extract the reflected beam outside the confines of the collimated beam, the distance to the focus (the length of the beam after the mirror) must exceed the radius of this collimated beam. At the same time, the minimum distance between the aperture stop and the focus is limited only by the thickness of the optical element in which it is made. Thus, the aperture size and the losses owing to shading will be lower than for a supplementary mirror. In order to avoid the technically complicated making of apertures in the diffraction grating or the objectives, the plane mirrors 3 and 10 have been used in the proposed configuration. They "break" the optical axis as they direct the collimated light beams at the diffraction grating and objectives, and they have aperture stops through which the light enters and leaves the system.

During their forward and reverse passage, part of the collimated beams illuminate the detector through the wide aperture stops. To eliminate this, two light-absorbing screens are mounted near the collimator mirror 4 and plane mirror 10. Screen 5 blocks illumination of the detector by the entering light beam after collimation and screen 8 , after dispersion subtraction. Positioning the screen near the plane mirror 10 ensures minimal masking of the useful light flux and is more efficient than a separate screen near the diffraction grating. Instead of this screen, the aperture stop in mirror 10 can be widened or a light-absorbing coating can be used.

Results of Numerical Simulations. The simulations used the optical design package ZEMAX(R) in sequential and nonsequential ray tracing modes. Studies were made of the propagation of light beams with a nominal numerical aperture of 0.11 from points in the center, corners, and midpoints of the sides of the entrance aperture. The aperture stop of the system was mounted on the collimator objective; in a real configuration its location may be different because of the effect of the light collection system.

When modelling narrow-band spectral filtration it is important to confirm the correctness of the superposition of the images in different wavelengths after dispersion subtraction (i.e., that there is no color smearing). The polychromatic light was simulated by a discrete set of wavelengths specified with a step size of $4 \mathrm{~nm}$ over $480-520 \mathrm{~nm}$ (the $500 \mathrm{~nm}$ beam 


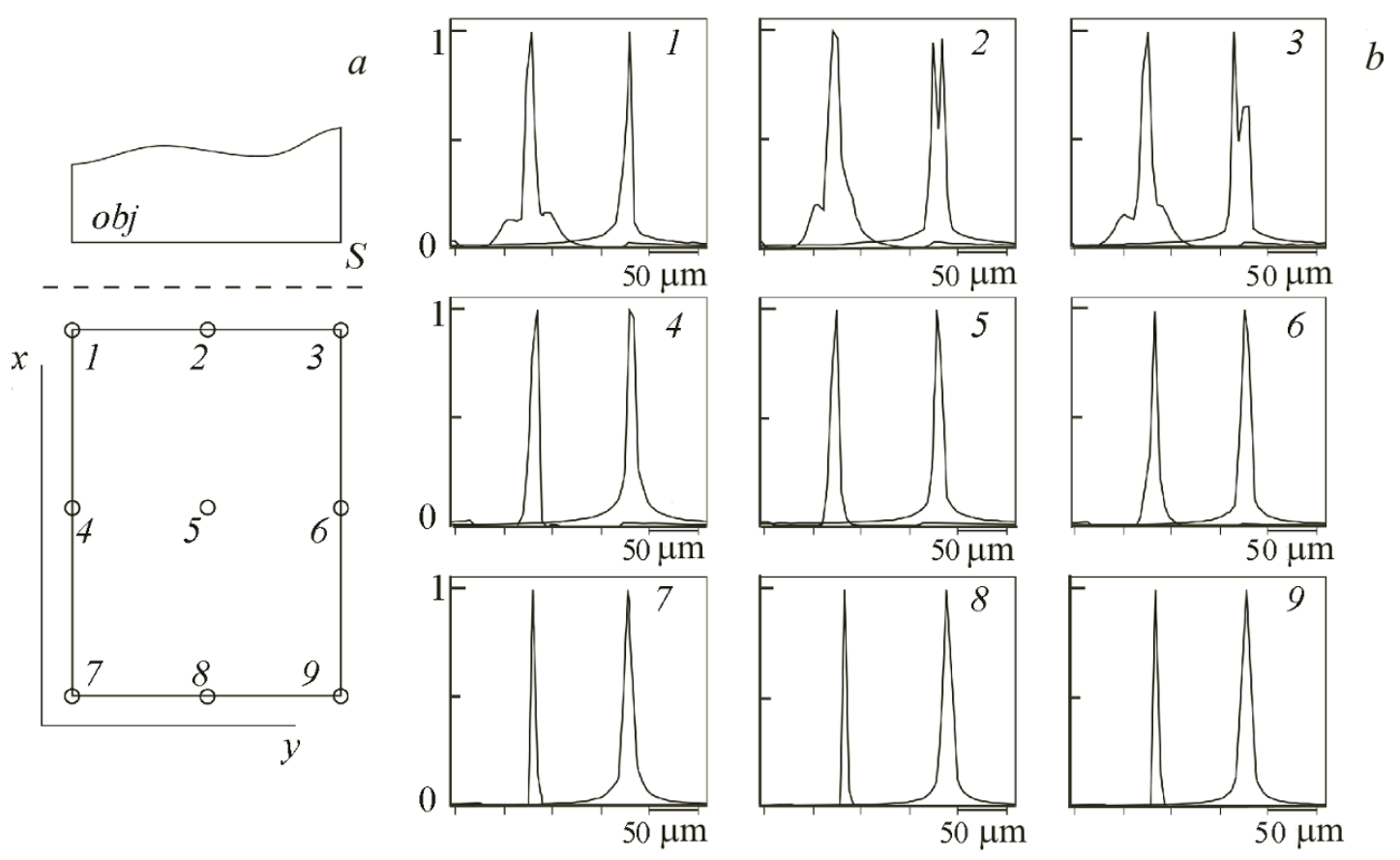

Fig. 2. The shape of the detector, symmetry plane $S$, and edge of the entrance aperture obj projected onto the plane of the exit aperture of the imaging monochromator (a) and the cross sections of the scattering spots at the characteristic spots of the detector (b); for each point the left profile corresponds to the $\mathrm{x}$ direction and the right profile, to the y direction (the dispersion direction); the characteristic points 1,3, 7, and 9 are attached to corners; $2,4,6$, and 8 are attached to the centers of the sides; and 5 is attached to the center of the exit aperture (detector).

from the center of the entrance aperture passes through the center of the slit mask 13). Thus, in the following, the term "scattering spot" refers to the image of a point of the entrance aperture on the detector formed by the superposition of the chromatic components passing through the aperture in the slit mask. Optimum focussing of the image plane was defined in terms of the criteria of a minimal half width of the profiles and maximum uniformity of the spot sizes over the field (image plane). Attainment of spectral resolution at a level of $10 \mathrm{~nm}$ was specified for the model; this raised the problem of attaining maximum spatial resolution for a sufficiently large entrance aperture (i.e., getting as large a number as possible of spatially resolveable elements in the frame), first by minimizing the angles at which the light beams pass through the objective. The values of the configuration parameters given below have been chosen from a multitude of variants.

Profiles of the scattering spots calculated in a sequential tracing mode are shown in Fig. 2. They are mostly close to symmetric (the asymmetry owing to off-axis aberrations is small) and have faint, narrow "wings." However, the profiles are distorted in some regions (mainly near the symmetry plane S, points 1-3): the intensity is reduced in the center and the "wings" have stepped shape. Since a longitudinal displacement of the detector from its optimal position does not reduce the size of the scatter spots, the intensity dips in the scattering spots appear to be caused by light loss owing to the aperture stops and shading of the region of the light beam by the light-absorbing screens, rather than by focussing errors.

The differences in the profiles of the spots with distance from the symmetry plane are probably explained by an increased aberration contribution from the large angle of incidence of the light beams as the source points approach the symmetry plane: the maximum angles at which the beams pass from points 1-3 through the mirror objectives are estimated to exceed the analogous angles for the beams from points $4-9$ by at least $20 \%$. This explanation is supported by the more distinct aberration component (asymmetry) in the scattering profiles for points 1-3. The existence of residual aberrations in the system is indicated by the asymmetry in the profiles relative to the symmetry plane parallel to the $\mathrm{x}$ axis (points 4 and 6 , 7 and 9). The asymmetry in the beam passage through the objectives in the meridional and sagittal cross sections shows up in the different configurations of the profiles along the $x$ and $y$ directions. 
TABLE 1. Profile Half Width of the Scatter Point for Several Points of the Entrance Field

\begin{tabular}{|c|c|c|c|c|c|c|}
\hline \multirow{2}{*}{$\begin{array}{c}\text { Point No. } \\
\text { (see Fig. 2) }\end{array}$} & \multicolumn{2}{|c|}{ Coordinate at entrance aperture, $\mathrm{mm}$} & Coordinate at exit aperture, $\mathrm{mm}$ & \multicolumn{3}{|c|}{ Profile half width, $\mathrm{mm}$} \\
\cline { 2 - 7 } & $x$ & $y$ & $x$ & $y$ & $x$ & $y$ \\
\hline 1 & 1.33 & 2.67 & 1.41 & 2.29 & 9 & 7 \\
3 & 1.33 & 0 & 1.41 & -0.33 & 13 & 16 \\
4 & 1.33 & -2.67 & 1.41 & -2.96 & 10 & 19 \\
5 & 4.92 & 2.67 & 4.92 & 2.29 & 9 & 9 \\
6 & 4.92 & 0 & 4.92 & -0.33 & 8 & 9 \\
7 & 4.92 & -2.67 & 4.92 & -2.95 & 7 & 11 \\
8 & 8.51 & 2.67 & 8.44 & 2.28 & 5 & 9 \\
9 & 8.51 & 0 & 8.45 & -0.33 & 5 & 10 \\
\hline
\end{tabular}

Note. The directions $x$ and $y$ correspond to Fig. 2 ( $y$ is parallel to the dispersion direction).

A procedure for estimating the half width of the scattering spots was developed which takes the pixellation of the photodetector into account: the beam tracing results in the optical design program are summed pixel by pixel and are interpolated when necessary. The data shown here were obtained for pixel sizes of $5 \times 5 \mu \mathrm{m}$ and an interpolation step size of 1 $\mu \mathrm{m}$ with an error of $\pm 1 \mu \mathrm{m}$. The typical half widths do not exceed $10 \mu \mathrm{m}$, except for points 2 and 2 for which the coordinates at the half width give $15-20 \mu \mathrm{m}$ (Table 1).

The estimated effective number of resolved spatial elements is $\sim 4 \times 10^{5}$ on a $7.18 \times 5.32 \mathrm{~mm}$ array. The simulations confirmed a theoretical estimate [15] of the width of an isolated spectral band for a point on the object. For an aperture width of $6.7 \mathrm{~mm}$ in the mask, it is equal to $15.6 \mathrm{~nm}$ (theoretically calculated at $16.6 \mathrm{~nm}$ ), and for a width of $1 \mathrm{~mm}, 2.4 \mathrm{~nm}$ (theoretical $2.5 \mathrm{~nm}$ ).

The sources of background illumination of the detector through the aperture stops were evaluated by simulation in the nonsequential tracing mode. It was found that the bulk of the illumination is caused by the beams after first reflection from the collimator objective, as well as by the beams after dispersion subtraction during reverse propagation. Two lightabsorbing screens are enough to stop these beams (Fig. 1): a rectangle 5 of size $13.6 \times 10.8 \mathrm{~mm}$ located near the center of the objective 4 and a rectangle 8 of size $6 \times 18.4 \mathrm{~mm}$ located above the aperture stop of the plane mirror 10 . In designing the optical configuration it should be kept in mind that the position of the screen 5 depends on the passage of light beams from the entrance aperture and when pre-optics are used this arrangement will be different.

The aperture stops and shading from light-absorbing elements reduce the effective optical throughput of the system. It was estimated that for a nominal numerical aperture of 0.11 the area losses for the light beam are $\leq 21 \%$, which is equivalent to the actual numerical aperture of $\geq 0.087$. For different points in the field the aperture differs, and this value is valid for points in the field with the smallest mutual overlap of the shaded regions (Fig. 2, points 1-3). For the points with the largest overlap (smaller mutual shading) the light losses decrease to $19 \%$, which corresponds to an actual numerical aperture of 0.09 (points 4-6). Further from the symmetry plane (points 7-9) the losses increase, but do not reach the levels of points 1-3. It should be emphasized that this estimate of the losses is generally high. The losses can be reduced (e.g., by increasing the sizes of the optical elements or nominal aperture while maintaining the aperture and detector dimensions), and the aperture stops can be profiled in the planes of the mirrors over the cross section of the light beam.

Conclusions. Numerical simulations of the proposed imaging monochromator configuration with dispersion subtraction has shown that the off-axis aberrations are reduced compared to the configuration of [14] because the light beam propagation is closer to axially symmetric. The resulting characteristics (an entrance window of size $7.18 \times 5.32 \mathrm{~mm}$ yields a typical scattering spot size of $\sim 10 \mu \mathrm{m}$ and an effective numerical aperture of $\sim 0.09$, while the spectral transmission bandwidth is determined by the width of the aperture in the slit mask, e.g., $\sim 2.4 \mathrm{~nm}$ for an aperture width of $1 \mathrm{~mm}$ ) indicate that this configuration can be used as a basis for constructing an imaging monochromator. This instrument can be used for multispectral scanning. With a working bandwidth corresponding to the visible spectrum, the number of spectral channels, equal to the ratio of that $400 \mathrm{~nm}$ range to the transmission bandwidth, is 160 for an aperture width of $1 \mathrm{~mm}$ in the mask. The 
time required to record a multispectral image in all channels is limited by the time for realigning the dispersing element (in the existing prototype spectral scan system, the time to change the channel is $1 \mathrm{~s}$ ). The availability of efficient components (spherical objectives, plane mirrors, a plane reflecting diffraction grating) means that this design offers promise for large scale multispectral scanning applications.

\section{REFERENCES}

1. P. J. Curran, Int. J. Appl. Earth. Observ. Geoinform., 3, No. 4, 305-312 (2001).

2. Yichun Xie, Zongyao Sha, and Mei Yu, J. Plant. Ecol., 1, No. 1, 9-23 (2008).

3. M. Herold and D. A. Roberts, Int. J. Geoinform., 2, No. 1, 1-13 (2006).

4. M. Borengasser, W. Hungate, and R. Watkins, Hyperspectral Remote Sensing: Principles and Applications, Boca Raton, CRC Press (2007), pp. 63-111.

5. N. Hagen, R. Kester, L. Gao, and T. Tkaczyk, Opt. Eng., 51, No. 11, 111702-1-111702-7 (2012).

6. P. Mouroulis, R. O. Green, and T. G. Chrien, Appl. Opt., 39, No. 13, 2210-2220 (2000).

7. E. S. Voropai, I. M. Gulis, A. G. Kupreyeu, K. N. Kaplevskii, A. G. Kostyukevich, A. E. Rad'ko, and K. A. Shevchenko, Zh. Prikl. Spektrosk., 77, No. 2, 305-312 (2010).

8. N. Gat, Proc. SPIE, 4056, 50-64 (2000).

9. D. Ren and J. Allington-Smith, Publ. Astron. Soc. Pac., 114, No. 798, 866-878 (2002).

10. M. E. Gehm, R. John, D. J. Brady, R. M. Willett, and T. J. Schulz, Opt. Express, 15, No. 21, 14013-14027 (2007).

11. L. Gao, R. T. Kester, N. Hagen, and T. S. Tkaczyk, Opt. Express, 18, No. 14, 14330-14344 (2010).

12. D. L. Gilblom and S. K. Yoo, Proc. SPIE, 5301, 186-192 (2004).

13. B. L. Markham, P. W. Dabney, J. E. Murphy-Morris, and J. A. Pedelty, IEEE International, 25-30 July 2010, Honolulu, HI (2010), pp. 2283-2286.

14. I. M. Gulis A. G. Kupreyeu, and A. G. Kostyukevich, Vestn. Bel. Gos. Univ., Ser. 1, No. 2, pp. 19-23 (2011).

15. I. M. Gulis and A. G. Kupreyeu, Vestn. Bel. Gos. Univ., Ser. 1, No. 3, 3-7 (2014).

16. W. T. Welford, Aberrations of Optical Systems, McGraw-Hill, Bristol (1989), pp. 92-129.

17. I. M. Gulis and A. G. Kupreyeu, Vestn. Bel. Gos. Univ., Ser. 1, No. 3, pp. 11-15 (2008). 